Revue de droit comparé du travail et de la sécurité sociale

3 | 2017

Le travail dans l'économie informelle, un défi pour le droit social

\title{
Incorporer et formaliser l'emploi informel en Chine
}

Dan Wei

\section{OpenEdition}

Journals

Édition électronique

URL : https://journals.openedition.org/rdctss/380

DOI : $10.4000 /$ rdctss.380

ISSN : 2262-9815

Éditeur

Centre de droit comparé du travail et de la sécurité sociale

Édition imprimée

Date de publication : 1 septembre 2017

Pagination : 116-127

ISSN : $2117-4350$

\section{Référence électronique}

Dan Wei, « Incorporer et formaliser l'emploi informel en Chine », Revue de droit comparé du travail et de la sécurité sociale [En ligne], 3 | 2017, mis en ligne le 01 novembre 2018, consulté le 04 décembre 2021. URL : http://journals.openedition.org/rdctss/380 ; DOI : https://doi.org/10.4000/rdctss.380

\section{(ब) $\Theta \Theta$}

Revue de droit comparé du travail et de la sécurité sociale est mise à disposition selon les termes de la Licence Creative Commons Attribution - Pas d'Utilisation Commerciale - Pas de Modification 4.0 International. 


\section{INCORPORER ET FORMALISER L'EMPLOI informel EN CHINE}

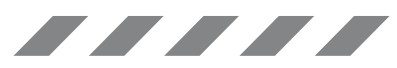

\section{ABSTRACT}

The paper describes the changing scenario of informal employment in China, analyzes its conception and composition of informal workers, and scrutinizes China's approach aimed at incorporate and formalize the informal employment, the existing legal tools and the shifting government's policies. The author tries to summarize the experiences in the past and point out some possible trends in the future.

KEY WORDS : China, Informal employment, labor contract, employment relationship, circumvention.

\section{RÉSUMÉ}

Cet article s'intéresse à la situation de l'emploi informel en Chine. Il propose un examen approfondi des initiatives engagées au niveau national visant à intégrer et formaliser l'emploi informel, des outils juridiques existants et de l'évolution des politiques gouvernementales. Nous tenterons donc de rendre compte des expériences du passé et de présenter quelques tendances futures potentielles.

\section{MOTS CLÉS: Chine, emploi informel, contrat de travail, relation d'emploi, contournement.}


usqu'à la fin des années 1970, l'emploi informel n'existait pas en Chine, car la quasitotalité des travailleurs était absorbée par les secteurs formels traditionnels de l'économie nationale ou de la propriété collective, dans le cadre de l'économie planifiée. A partir de 1978, lorsque les réformes économiques ont été lancées, la Chine a connu un processus d'informalisation rapide de son modèle d'emploi. Plus précisément, lorsque l'économie de marché socialiste a été officiellement lancée par Amendement à la Constitution, en 1993, puis par la réforme tendant à privatiser les entreprises publiques, des millions de travailleurs ont été licenciés des entreprises publiques ou des établissements en propriété collective. En même temps, en raison de l'accélération du processus d'urbanisation, au moins 160 millions de travailleurs ruraux ont migré vers les zones urbaine ${ }^{01}$. A défaut de statistiques officielles sur l'emploi informel en Chine, il faut se fier aux rapports existants qui font état de chiffres approximatifs. Entre 1990 et 2010, le nombre de travailleurs informels a augmenté, passant de 28,94 millions à 219 millions, ce qui représente respectivement $17,5 \%$ et $63,2 \%$ du marché du travail, soit un taux de croissance annuel de $6,3 \%{ }^{02}$. En 2006, le nombre de travailleurs informels avoisinait les 130 millions $^{03}$, pour se porter à 190 millions, en 200904. En 2015, il y avait environ 330 millions de travailleurs informels en Chine, ce qui correspond à près de $75 \%$ de la population active urbaine ${ }^{05}$.

En Chine, tout comme dans d'autres économies en voie de développement et émergentes, l'emploi informel est devenu la source la plus importante de l'emploi urbain et de la création d'emplois. Le développement rapide de l'emploi informel peut être attribué aux raisons suivantes.

Premièrement, au cours des dernières décennies, la structure économique de la Chine a été transformée. Le nombre de travailleurs du secteur tertiaire a connu une croissance bien plus rapide que celui des secteurs manufacturier et agricole. La compétitivité des entreprises publiques s'est effondrée tandis que le secteur non public de l'économie (dont le secteur privé et les travailleurs indépendants) s'est avéré plus dynamique et a donc pu offrir davantage d'opportunités d'emploi. Un grand nombre de travailleurs, salariés dans les secteurs manufacturier, de l'extraction et de la construction, se sont tournés vers le secteur informel, et le nombre de salariés des sociétés anonymes coopératives, des entreprises à capitaux étrangers et des entreprises privées n'a cessé d'augmenter.

01 J. Wang, F. Lee Cook and Z. Lin, « Informal Employment in China : Recent Development and Human Resource Implications », Asian Pacific Journal of Human Resources, n54, 2016, p. 295.

02 Task Group of Labor Relations Institute of China, « New Characteristics and Counter measures of Informal Employment Growth in China » (en chinois), Economic Review, 1, $2 n^{\circ} 013$, p. 57-58.

03 Y. Ren and X. Peng, « 2006 China Informal Employment Development Report: Re-observation of the Labor Market » (en chinois), Chongqing, Chongqing Publishing Group, 2006, Pour plus d'informations, voir: http:// society.people.com.cn/GB/6680745.html.

04 F. Chen, «Informal Economy and Chinese Model of Urbanization » (en chinois), 2015 http://www.cwzg.cn/ theory/201509/24714.html.

05 Z. Huang, « Reconsideration of China's Informal Economy: An Introduction from the Perspective of Social and Economic History and Legal History » (en chinois),2017, http://fashi.ecupl.edu.cn/s/316/t/107/6e/6b/ info28267.htm 
Deuxièmement, l'économie chinoise est un modèle à fort coefficient de main-d'œuvre, avec un excédent de travailleurs ruraux, non qualifiés ou semi-qualifiés. L'offre a toujours été plus forte que la demande sur le marché du travail. Contrairement aux emplois formels, les emplois informels paraissent plus accessibles et adaptés à cette main-d'œuvre excédentaire.

Troisièmement, comme première économie en transition et deuxième économie mondiale, l'essor de l'économie de marché et la libéralisation ont en outre impliqué l'abaissement du coût du travail et l'accroissement de la productivité des travailleurs. Parce qu'il permet d'absorber une partie de cette main-d'œuvre tout en offrant davantage de flexibilité, l'emploi informel a fait l'objet d'attention et de soutien de la part des organismes gouvernementaux.

Enfin, ces dernières années, on observe une nouvelle tendance : à côté des travailleurs non qualifiés, un nombre sans cesse croissant de travailleurs très qualifiés éprouvent des difficultés à trouver un emploi (surnommés « la tribu des fourmis » en raison de leurs faibles salaires et de leurs domiciles éloignés du centre des grandes villes). Ces travailleurs se tournent aussi vers le marché du travail informel. En raison de la stratégie de développement de l'enseignement supérieur et des embauches permanentes dans l'enseignement, le nombre de diplômés universitaires est passé de 2 millions à 7 millions entre 2003 et $2015^{06}$. On assiste également à l'émergence de très petites entreprises et d'entreprises à domicile, avec des personnes qui préfèrent devenir travailleurs indépendants et s'engager dans l'ecommerce ou d'autres activités en ligne du même genre ${ }^{07}$.

\section{I - Conception de l'emploi informel et typologie des travailleurs informels}

Avant 2016, le terme « emploi informel » ne figurait dans aucun document officiel en Chine. Les organismes gouvernementaux ont recouru à la distinction entre " emploi flexible » et « emploi formel ». Dans une certaine mesure, «l'emploi flexible », auquel les documents officiels font référence, présente des caractéristiques similaires à celles de « l'emploi informel $»^{08}$. C'est pourquoi on ne dispose d'aucune statistique officielle sur «l'emploi informel».

Il n'existe toujours pas à l'heure actuelle de définition de l'emploi informel faisant consensus en Chine. Dans la littérature, la conception de l'emploi informel retenue par les universitaires chinois ${ }^{09}$ est différente de celle en vigueur dans les pays développés, qui ont

06 J. Wang, F. Lee Cook and Z. Lin, « Informal Employment in China: Recent Development and Human Resource Implications », Asian Pacific Journal of Human Resources, n $.54,2016$, p. 295.

07 B. Dong, « Non-Standardized Labor Relations » (en chinois), Academic Research, n7, 2008, p. 55.

08 Information Office of the State Council of the People's Republic of China, « China's Employment Situation and Policies », 2004, part II, http://www.chinadaily.com.cn/english/doc/2004-04/26/ content 326356.htm.

09 Voir par exemple A. Hu and Y. Yang, « Transformation of Employment Patterns: From Formalization to Informalization » (en chinois), Management World, n², 2001, p. 69-78; J. Wang, F. Lee Cook and Z. Lin, « Informal Employment in China: Recent Development and Human Resource Implications », Asian Pacific Journal of Human Resources, n54, 2016, p. 292-311; Z. Liang, S. Appleton and L. Song, « Informal Employment in China: Trends, Patterns and Determinants of Entry », IZA Discussion Paper, $n^{\circ}$ 10139, August 2016, p. 1-23; Y. Zhang, « Protection of Labor Rights in Informal Sectors » (en 
opté pour un modèle centré sur l'activité. Les universitaires chinois semblent, pour leur part, privilégier une approche centrée soit sur l'entreprise soit sur les emplois ${ }^{10}$. En Chine, l'emploi informel englobe des emplois aussi bien des secteurs informels que formels. En effet, pour relever du secteur informel, les travailleurs doivent : être indépendants (des personnes ou des sociétés en nom collectif) ; être employés dans des services indépendants (comme domestiques, vendeurs ambulants, ou aidants temporaires dans les hôpitaux payés à l'heure) ; travailler pour de petites et microentreprises; ou encore travailler en free-lance lcomme les avocats, les écrivains, les traducteurs et d'autres salariés d'agences de services). Pour ce qui est du secteur formel, les travailleurs doivent : être employés par de moyennes ou grandes entreprises ly compris dans le cadre de relations de travail atypiques comme les travailleurs temporaires, les saisonniers, les intérimaires, les travailleurs détachés ${ }^{11}$, les employés de services et les ouvriers payés à l'heure) ; être employés par des organisations du travail ( y compris le travail détaché ou temporaire) ; ou être employés par les gouvernements disposant de subventions (comme les services des gouvernements municipaux, les communautés locales ou les organismes du secteur public) ${ }^{12}$.

En termes de répartition géographique, on observe une tendance générale selon laquelle les travailleurs des régions de la côte sud-est, où l'économie de marché connaît un fort essor, prennent habituellement l'initiative de chercher des opportunités de travail informel ; à l'opposé les travailleurs des régions du nord-est, où le taux de chômage est élevé, se voient obligés d'accepter l'emploi informel comme moyen de subsistance ${ }^{13}$.

Malgré une répartition sectorielle très large, l'emploi informel prévaut dans les secteurs informels et le secteur tertiaire, d'une manière générale sous la forme d'emplois de courte durée, temporaires, à temps partiel, en détachement, indépendants ou domestiques. Même si un certain nombre de travailleurs informels disposent d'une bonne formation et ont des compétences professionnelles, la plupart d'entre eux viennent des zones rurales, n'ont pas les compétences suffisantes, n'ont pas reçu de formation adaptée et sont plus vulnérables dans les relations de travail complexes, flexibles et instables. Les travailleurs informels, au sens large,

chinois), Journal of China Institute of Industrial Relations, n²2(1), February 2008, p. 43-46; Ping He and Yingfang Hua, «Study on Social Security of Informal Employment » (en chinois), Beijing, China Labor and Social Security Publishing House, 2008.

10 C. C. Williams and M. A. Lansky, «Informal Employment in Developed and Developing Economies: Perspectives and Policy Responses », International Labour Review, Vol. 152, 2013, n³-4, p. 356-358. See also R. Hussmanns, « Defining and measuring informal employment" https://pdfs.semanticscholar.org/1 4ba/9f7951a6ef3be56b2fdf39953ecf5989f085.pdf.

11 Le travail détaché est devenu une forme courante d'emploi en Chine. Les travailleurs peuvent être mis à disposition dans toute entité par une agence de travail en détachement. Cette agence doit conclure un contrat avec le travailleur détaché, d'une durée minimum de deux ans. Dans le contrat de travail doivent être indiqués, la durée du contrat l'entreprise dans laquelle le travailleur exercera son activité, les conditions de détachement, le poste de travail, etc. L'agence conclut des conventions avec les entreprises utilisant les travailleurs détachés concernant les postes de travail, le nombre de travailleurs concernés, le montant et les conditions de de la rémunération ainsi que les questions relatives à la sécurité sociale. En Chine, le travail détaché peut être transrégional et intersectoriel. Voir les articles 57-67 de la loi chinoise sur le contrôle du travail (2008, amendée en 2012) et les dispositions provisoires de 2014 sur le détachement du personnel.

12 J. Wang, F. Lee Cook and Z. Lin, « Informal Employment in China: Recent Development and Human Resource Implications », Asian Pacific Journal of Human Resources, n54, 2016, p. 297.

13 A. Hu and Y. Yang, «Transformation of Employment Patterns: From Formalization to Informalization » (en chinois), Management World, ${ }^{\circ} 2,2001$, p. 74 
ne connaissent pas la stabilité de l'emploi et ne peuvent pas bénéficier de la sécurité sociale de base ni de la protection du droit du travail, en particulier des avantages statutaires et non statutaires. En comparaison avec les emplois formels, la rémunération des emplois informels est bien plus faible, à l'exception des professionnels indépendants à hauts revenus qui sont à leur propre compte. En raison de facteurs culturels traditionnels, la reconnaissance et la valeur sociale de certains types d'emploi (ex. aides domestiques) sont aisément sous-évaluées. Comme travailleurs informels, les femmes, les travailleurs migrants et les personnes atteintes de handicap sont plus susceptibles de subir des discriminations.

La nature informelle des relations d'emploi peut entraîner un manque de confiance mutuelle entre l'employeur et les travailleurs. La vulnérabilité des travailleurs informels est en outre intensifiée par l'absence de relations de travail durables, l'insuffisance des réglementations et l'offre excédentaire de travailleurs sur le marché chinois.

\section{II - Limites des solutions juridiques concernant l'emploi informel}

Le cadre juridique du droit du travail chinois comprend à la fois des instruments internationaux et des règlementations nationales. La Chine a ratifié plusieurs conventions de l'OIT : la Convention sur l'âge minimum, celles sur l'interdiction des pires formes de travail des enfants, sur l'égalité de rémunération, sur la politique de l'emploi. Les lois et règlementations nationales principales comprennent la Loi sur les syndicats (1992, amendée en 2001), la Loi sur le travail (1995, amendée en 2009), la Loi sur la sécurité au travail ( 2002), la Loi sur la prévention et le contrôle des maladies professionnelles (2002), la Loi sur le contrat de travail (2008, amendée en 2012), la Loi sur le règlement des litiges et l'arbitrage (2008), la Loi de promotion de l'emploi (2008, amendée en 2015), la Loi sur la sécurité sociale (2011), la Règlementation sur le salaire minimum en entreprise (2004), la Règlementation sur la mise en œuvre de la Loi sur le contrat de travail (2008), la Règlementation provisoire sur les services de détachement du personnel (2014), les Avis sur la construction de relations professionnelles harmonieuses (2015), et un certain nombre de normes promulguées par le Ministère des Ressources humaines et de la sécurité sociale, etc.

Lorsqu'on évalue l'efficacité globale des solutions juridiques existantes, on découvre que la couverture contractuelle des migrants a nettement augmenté, accompagnée par l'accès aux assurances sociales et à d'autres prestations ${ }^{14}$. Cependant, la majorité des travailleurs sont employés de manière informelle. De nombreux vides juridiques restent donc à combler, et l'application effective des textes demeure un défi majeur à relever.

Si la Loi sur le travail comprend des dispositions élémentaires, dont l'obligation d'établir un contrat de travail pour protéger les droits des travailleurs, moins de $20 \%$ des petites et moyennes entreprises ont conclu des contrats de travail avec leurs salariés, et les entreprises individuelles sont encore moins nombreuses à introduire des contrats

14 R. Freeman and X. Li, « Has China's new labour contract worked?"and Zhiming Cheng, Russell Smyth and Fei Guo, "The Impact of China's New Labour Contract Law on Socioeconomic Outcomes for Migrant and Urban Workers », Monash University, Department of Economics. Discussion Papers, 51/13, 2013, p.1-31 
de travail ${ }^{15}$. La Loi sur le contrat de travail a été promulguée pour renforcer les dispositions contractuelles et pour mieux protéger les droits et intérêts légitimes des salariés, en clarifiant les droits et obligations des deux parties à la relation de travail ${ }^{16}$.

Dans l'objectif de combler les vides juridiques laissés par la Loi sur le travail, en complément des contrats traditionnels, la Loi sur le contrat de travail prévoit également des dispositions pour les contrats moins classiques, à savoir ceux établis dans le cadre d'un détachement (principalement au moyen de l'amendement en 2012) et pour les emplois à temps partiel. Le législateur a adopté différentes approches pour réglementer ces deux aspects, le travail dans le cadre d'un détachement faisant l'objet d'une approche stricte et inflexible tandis que les emplois à temps partiel font l'objet d'une approche beaucoup plus indulgente et souple ${ }^{17}$.

La Loi sur le contrat de travail pose un certain nombre d'exigences en matière de recours au travail détaché : les droits et obligations des prestataires de service, des travailleurs et des entreprises d'accueil ; la conclusion de contrats de détachement, etc. Ces dispositions montrent que le législateur a bien essayé de protéger les intérêts des travailleurs détachés en tant que travailleurs informels et, par ricochet, de formaliser l'emploi informel. Ainsi, durant une période sans travail, le prestataire de services devra verser une compensation mensuelle aux travailleurs détachés, basée sur le salaire minimum prescrit par le gouvernement populaire du lieu où le prestataire de services est domicilié ${ }^{18}$. En outre, les travailleurs détachés ont le droit de recevoir le même salaire que celui perçu par les salariés de l'entreprise utilisatrice pour un même travail ${ }^{19}$.

Pour ce qui est des emplois à temps partiel qui sont rémunérés à l'heure, et pour lesquels la durée moyenne de travail est inférieure ou égale à 4 heures par jour ou 24 heures par semaine ${ }^{20}$, il existe des règles obligatoires. Par exemple, le calcul de la rémunération du travail à temps partiel, sur une base horaire ne sera pas inférieur au salaire horaire minimum prescrit par le gouvernement populaire du lieu où l'employeur est domicilié. Le délai de paiement maximum et la fréquence de paiement des salaires des travailleurs à temps partiel ne seront pas supérieurs à 15 jours $^{21}$. En même temps, la Loi sur le contrat de travail affirme la flexibilité de l'emploi informel, en reconnaissant la faculté aux deux parties à la relation de travail de conclure un accord oral ${ }^{22}$. L'emploi à temps partiel ne fait pas l'objet d'une période d'essai ${ }^{23}$. De plus, l'une ou l'autre partie peut informer l'autre de la résiliation du contrat à tout moment. Lors de la résiliation d'un emploi à temps partiel,

15 L. Mao and W. Du, « Why to Enact the Labor Contract Law with the Labor Law already in Force? » (en chinois), People's Daily, March 29, 2006, http://www.lawfirm.com.cn/Subject/SubjectNews info. asp?!d=14.

16 Article 1 de la Loi chinoise sur le contrat de travail (2008, amendée en 2012).

17 L. Li, «Retrospect and Prospect of Non-standard Labor Legislations of China » (en chinois), Labour Unions Studies, No. 5, 2014, p. 17. Voir aussi C. Xin ed., «Annotation of Labor Contract Law of People's Republic of China » (en chinois), Beijing, Law Press China, 2013, p.290.

18 Article 58 de la Loi chinoise sur le contrat de travail (2008, amendée en 2012).

19 Article 63 de la Loi chinoise sur le contrat de travail (2008, amendée en 2012).

20 Article 68 de la Loi chinoise sur le contrat de travail (2008, amendée en 2012).

21 Article 72 de la Loi chinoise sur le contrat de travail (2008, amendée en 2012).

22 Article 69 de la Loi chinoise sur le contrat de travail (2008, amendée en 2012).

23 Article 70 de la Loi chinoise sur le contrat de travail (2008, amendée en 2012). 
l'employeur ne verse aucune compensation économique au salarié24. Jusqu'à ce jour, aucune loi ou règlement administratif national n'a traité des assurances obligatoires pour les travailleurs à temps partiel.

Malgré ces efforts législatifs, il existe toujours d'importantes questions à résoudre. Tout d'abord, l'absence d'une définition claire du «travail » dans le droit du travail est une entrave à une protection adéquate des travailleurs informels. A ce jour, la définition très étroite du « travail » par le droit du travail fait référence à des personnes physiques qui se trouvent dans une relation de travail avec cinq types d'employeurs (entreprises, organisations économiques individuelles, services de l'Etat, organisations institutionnelles et groupes sociaux) ${ }^{25}$. La Loi sur le contrat de travail a ajouté les « entités privées non commerciales » dans la catégorie des employeurs ${ }^{26}$. Néanmoins, un certain nombre de travailleurs informels ne sont toujours pas considérés comme de vrais «travailleurs » par les lois du travail en vigueur, comme les domestiques qui en sont statutairement exclus.

La relation juridique à laquelle donne lieu l'emploi informel constitue un exemple de zone grise car elle n'est ni reconnue ni interdite expressément par le droit du travail. Dans de nombreux cas, les formes de travail atypiques de l'emploi informel lcomme dans le cas des entrepreneurs indépendants, des travailleurs à temps partiel, des travailleurs temporaires recrutés par des agences de placement temporaire, ou par des entreprises de sous-traitance, des travailleurs occasionnels etc.) sont différentes des relations de travail classiques. Il peut être difficile de confirmer l'existence d'une vraie relation employeur/salarié ou de faire la distinction entre la relation d'emploi et la relation contractuelle établie par le biais du service de placement. D'autres fois, la relation d'emploi est ambigüe ou déguisée. La Loi sur le travail et la Loi sur le contrat de travail prétendent ajuster la relation d'emploi. Une fois de plus, la nature des relations de travail atypiques de l'économie informelle nécessiterait une redéfinition du travail.

En raison de l'ambigüité, des incertitudes ou des caractéristiques des dispositions légales en vigueur, le phénomène de contournement des lois du travail s'est aggravé en Chine. La Loi sur le contrat de travail révisée dispose ainsi que le travail détaché n'intervient qu'en complément des contrats de travail existants et s'applique exclusivement à des emplois provisoires, auxiliaires ou de remplacement ${ }^{27}$. Cependant, elle ne définit pas le type d'emplois considérés comme provisoires, auxiliaires ou de remplacement. Dans le même sens, certains employeurs peuvent délibérément opter pour la conclusion de contrats de travail à temps partiel plutôt qu'à temps plein pour s'exonérer de certaines obligations légales. Par ailleurs, au-delà de la qualification, la mise en œuvre des protections prévues par la législation peut poser problème. Par exemple, la Loi sur le travail prévoit un salaire égal pour un travail éga ${ }^{28}$ et la Loi sur le contrat de travail exige que les travailleurs détachés aient le droit de percevoir le même salaire que celui des salariés de l'entreprise effectuant le même travail ${ }^{29}$. Cependant, ces deux lois font l'impasse sur les modalités d'application de ce principe. Dès lors, le salaire égal pour un travail égal est difficile à

24 Article 71 de la Loi chinoise sur le contrat de travail (2008, amendée en 2012).

25 Article 2 du Droit du travail chinois (1995, amendé en 2009).

26 Article 2 de la Loi chinoise sur le contrat de travail (2008, amendée en 2012).

27 Article 66 de la Loi chinoise sur le contrat de travail (2008, amendée en 2012).

28 Article 46 du Droit du travail chinois (1995, amendé en 2009).

29 Article 63 de la Loi chinoise sur le contrat de travail (2008, amendée en 2012). 
mettre en place. Des salaires différents peuvent être justifiés en raison des missions confiées et des performances constatées sur un même poste de travail. Dans le but de réduire les coûts, d'augmenter au maximum les profits et d'éviter d'avoir recours aux relations d'emploi formelles, un certain nombre d'employeurs ont abusé ou détourné certaines dispositions légales, et cette «conformité créative» ${ }^{30}$ n'a engendré aucune responsabilité juridique. A ce titre, la promulgation de la Loi sur le contrat de travail n'est pas parvenue à atteindre les objectifs fixés à l'origine et a en outre stimulé le recours indu au travail détaché et à d'autres pratiques de contournement ${ }^{31}$. En conséquence, la situation des travailleurs informels ne s'est pas améliorée de manière significative.

La loi sur le travail reconnaît aux travailleurs huit principaux droits : le droit à l'égalité de traitement ; le droit de choisir librement sa profession ; le droit d'obtenir une rémunération ; le droit au repos, aux vacances et aux congés; le droit à la protection de la santé et à la sécurité au travail ; le droit à une formation professionnelle; le droit à la sécurité sociale ; et le droit d'action en vue de résoudre les conflits du travail. Parce qu'ils sont écartés ou parce que l'application des lois et des règlements est incomplète, certains de ces droits légaux ne sont pas garantis aux travailleurs informels. Les normes du travail relativement rigides, comme celles sur le salaire minimum, le temps de travail, les congés payés, le licenciement ou la sécurité sociale, ne s'appliquent pas de façon égale aux travailleurs informels. En réalité, ces dernierssubissent différents types de discrimination. Le droit au travail des travailleurs migrants et des femmes est toujours ignoré. Même si la Loi sur la sécurité sociale autorise plusieurs catégories de travailleurs (les ménages individuels, industriels et commerciaux sans salariés, les salariés à temps partiel et les autres personnes dont l'emploi est flexible) à participer aux assurances sociales de base et aux assurances médicales, ils sont tenus de payer toutes les cotisations eux-mêmes, de façon volontaire ${ }^{32}$, ce qui représente près de $20 \%$ de leurs salaires ${ }^{33}$. C'est pourquoi, dans les faits, un certain nombre de travailleurs informels en Chine nebénéficient d'aucune couverture de sécurité sociale.

Les travailleurs informels sont plus vulnérables en termes d'accès à la justice. Le système de recours actuel pour les travailleurs informels n'est pas efficace, pour diverses raisons. Tout d'abord, en cas de conflit du travail, les parties peuvent demander une médiation auprès du comité de médiation des conflits du travail de leur entreprise ; si le règlement ne peut aboutir par médiation et à la demande de l'une des parties, le conflit pourra faire l'objet d'un arbitrage devant un comité d'arbitrage des conflits du travail. Toute partie ayant des objections à formuler contre la décision du comité d'arbitrage du travail peut porter l'affaire devant un tribunal populaire ${ }^{34}$. L'arbitrage du travail est une procédure préalable à l'action en justice. Il n'est pas surprenant que les employeurs ne soient pas toujours prêts à accepter la médiation en cas

30 D. S. S. Cairns, « New Formalities for Casual Labor: Addressing Unintended Consequences of China's Labor Contract Law», Washington International Law Journal, Vol. 24, n¹, 2015, p. 235.

31 D. S. S. Cairns, « New Formalities for Casual Labor: Addressing Unintended Consequences of China's Labor Contract Law», Washington International Law Journal, Vol. 24, n²1, 2015, p. 232-236.

32 Articles 10 et 23 de la Loi sur la sécurité sociale (2011)..

33 Y. Ju, «A Legal Analysis of the Right and Interests of the Employees in Special Business » (en chinois), Journal of Hebei Normal University/Philosophy and Social Sciences Edition, Vol. 36, n², March 2013, p. 126. L'article 16 de la Loi sur la sécurité sociale (2011) dispose qu' « une personne relevant de l'assurance mixte de base devra recevoir une pension mensuelle de base à condition qu'il/elle ait cotisé durant au moins 15 ans au moment de l'âge légal de la retraite. »

34 Articles 77 et 79 de la Loi sur le travail (1995, amendée en 2009) et Article 5 de la Loi sur la médiation des conflits du travail et l'arbitrage (2008). 
d'emploi informel ou que les comités d'arbitrage du travail n'acceptent pas les demandes qui soulèvent des doutes concernant la nature des relations de travail. La vulnérabilité des travailleurs informels ne peut être protégée qu'au moyen d'une protection judiciaire. La recevabilité des affaires devant les tribunaux populaires dépend de l'existence de contrats de travail ou de relations d'emploi de fait.

Comme indiqué, certains travailleurs informels ne sont pas traités comme des « travailleurs », conformément aux règlementations du travail en vigueur lex. les entrepreneurs indépendants). Les relations de travail atypiques de l'économie informelle sont souvent perçues comme des relations contractuelles destinées à assurer la prestation de services entre des sujets égaux, et ne peuvent pas en conséquence être réglementées par les lois du travail. En raison des coûts élevés et de la longueur des procédures judiciaires, les travailleurs informels, partie la plus faible dans la relation, se retireront probablement après avoir été confrontés à un ensemble de difficultés. Enfin, il existe toujours énormément de barrières qui empêchent les travailleurs informels de faire appel au recours collectif. Jusqu'ici, les actions collectives se sont uniquement limitées aux cas de pollution environnementale et d'atteinte aux droits et intérêts légitimes du consommateur ${ }^{35}$. A l'exception des travailleurs détachés, qui disposent du droit légal reconnu par la Loi sur le contrat de travail de rejoindre le syndicat de l'agence de prestations de services ou de l'entité d'accueil, ou de constituer des syndicats ${ }^{36}$, les syndicats ne peuvent guère accueillir la participation d'autres types de travailleurs informels ${ }^{37}$.

\section{III - Fluctuations des politiques gouvernementales en matière d'emploi informel}

En Chine, favoriser l'emploi flexible a été une politique nationale importante pour accélérer la transformation des modèles d'emploi et pour atténuer les tensions professionnelles. Depuis les années 1980, les gouvernements, aussi bien au niveau central que local, ont défendu le développement de ce type d'emplois. Ainsi, selon le document "Politiques et situation de l'emploi en Chine », publié par le Bureau d'information du Conseil d'Etat en 2004, « de nouvelles formes d'emploi se sont multipliées, comme les emplois dans les sociétés à investissements étrangers et dans des entités économiques de formes diverses, les emplois à temps partiel, les emplois temporaires, les emplois saisonniers, le travail payé à l'heure et les emplois aux horaires flexibles; toutes ces nouvelles formes d'emploi ont largement contribué à la création d'emplois $»^{38}$. « Le gouvernement chinois encourage les travailleurs à rechercher un emploi parmi les diverses formes flexibles, et soutient activement la création d'organisations spécialisées dans le détachement du personnel pour assurer des services et une assistance en cas de recours à l'emploi flexible $»^{39}$.

35 Article 55 de la Loi de procédure civile de la Chine (adoptée en 1991 et révisée en 2007, 2012 et 2017).

36 Article 64 de la Loi chinoise sur le contrat de travail (2008, amendée en 2012).

37 L'article 3 de la Loi chinoise sur les syndicats (1992, amendée en 2001) prévoit que les travailleurs formels ont le droit de constituer et d'adhérer à des syndicats conformément à la loi.

38 http://www.chinadaily.com.cn/english/doc/2004-04/26/content_326356.htm.

39 http://www.chinadaily.com.cn/english/doc/2004-04/26/content_326356.htm. 
Il convient de mentionner que les organes locaux ont pris l'initiative de réglementer l'emploi informel. En 2003, pour la première fois, le Bureau du travail et de la sécurité sociale de Shanghai a adopté un document normatif totalement pionnier intitulé : «Pluralité d'opinions sur la standardisation des organisations d'emploi informel ${ }^{40}$. » Deux ans plus tard, en 2005, le Comité permanent du Congrès populaire de Shanghai a approuvé la première règlementation locale de toute la Chine, intitulée « Diverses dispositions pour la promotion de l'emploi à Shanghai $\gg^{41}$, qui visait à permettre la régularisation de l'emploi informel. D'autres municipalités (comme Tianjin et Chongqing) ont suivi l'exemple de Shanghai.

L'année 2008 marque le grand tournant des politiques nationales sur l'emploi informel. Avant 2008, l'attitude du gouvernement vis-à-vis de l'emploi informel était ambigüe, associant diverses mesures incohérentes d'encouragement, de restriction, d'acquiescement et de discrimination ${ }^{42}$. Cependant, après 2008, une approche plus cohérente accordant la priorité à la qualité de l'emploi plutôt qu'au nombre d'emplois créés a été adoptée. Actuellement, les efforts législatifs ont mis en avant la conceptualisation de la qualité de l'emploi, de la nondiscrimination, de la justice sociale, etc.

L'entrée en vigueur de la Loi de promotion de l'emploi (2008, amendée en 2015) clarifie le fait que les travailleurs ont droit à un emploi égal et à choisir un emploi de leur propre initiative $^{43}$. Elle mandate les syndicats pour protéger les droits des travailleurs ${ }^{44}$, donne instruction aux gouvernements à tous les niveaux de créer un environnement favorable à un emploi équitable, d'éliminer les discriminations en matière d'emploi, de formuler des politiques et de prendre des mesures pour soutenir et aider les personnes ayant des difficultés à trouver un emploi ${ }^{45}$. La Loi sur la sécurité sociale (2011) élargit la couverture de la sécurité sociale aux travailleurs informels ${ }^{46}$. L'amendement de la Loi sur le contrat de travail, en 2013, suivi par la Règlementation provisoire sur le travail détaché (2014), vise à corriger les abus en matière de détachement et à renforcer les règles applicables aux agences prestataires de services ${ }^{47}$. Les « Avis du Comité central du parti communiste chinois et du Conseil d'Etat sur la construction de relations de travail harmonieuses ${ }^{48}{ }^{\prime}$, en 2015 , ont mis l'accent sur l'importance de renforcer les droits de base des travailleurs migrants, des travailleurs détachés et d'autres travailleurs non conventionnels. Le très récent Plan d'action national chinois pour les droits de l'homme (2016-2020), annoncé par le Bureau d'information du Conseil d'Etat en septembre 2009, fixe des objectifs clairs pour améliorer la protection des travailleurs informels, «toute forme de discrimination en matière d'emploi étant strictement interdite. Des mesures seront prises pour traiter les arriérés de salaires

40 La version complète en chinois (téléchargée en 2095) est disponible sur http://www.12333sh.gov. $\mathrm{cn} / 201412333 / \mathrm{xxgk} / \mathrm{flfg} / \mathrm{gfxwj} / \mathrm{jygl} / \mathrm{fzg} / 201405 / \mathrm{t} 20140506$ 1181210.shtml.

41 La version complète en chinois est disponible sur http://www.12333sh.gov.cn/201412333/xwzx/ zxdt/201405/t20140504 1176736.shtml.

42 N. Zhao, « Non-standard Employment and Its Legal Promotion » (en chinois), Social Scientist, Supplement, Nov. 2007, p. 134.

43 Article 3 de la loi de promotion de l’emploi (2008, amendée en 2015).

44 Article 9 de la loi de promotion de l'emploi (2008, amendée en 2015).

45 Article 25 de la loi de promotion de l’emploi (2008, amendée en 2015).

46 Articles 10 et 23 de la Loi sur la sécurité sociale (2011).

47 R. Brown, "Chinese "Workers without Benefits" », Richmond Journal of Global Law \& Business, Vol. 15, 2016, p. 27.

48 Pour une version intégrale en chinois, voir http://news.xinhuanet.com/politics/2015-04/08/c 1114906835. htm. 
des travailleurs migrants, réglementer les licenciements, garantir les droits et intérêts des travailleurs employés de façon informelle, règlementer strictement l'application par les entreprises du système spécial des heures de travail, et renforcer le contrôle du travail $\gg^{49}$.

Pour conclure en Chine, l'emploi informel a connu un essor rapide et continuera à se développer en raison des caractéristiques de l'économie de transition, du marché national du travail et des impacts de l'économie de marché et de la libéralisation. Pendant de nombreuses années, le gouvernement a fait la promotion de l'emploi informel comme moyen d'absorption de la main-d'œuvre excédentaire. Cependant, la flexibilité excessive sur un marché du travail libéralisé n'entraîne pas automatiquement un développement socio-économique sain. L'efficacité économique sur le dos du bien-être et des avantages sociaux des travailleurs n'est pas durable à long terme. Les travailleurs informels, même s'ils représentent un pourcentage élevé de la main-d'œuvre dans son ensemble, font l'objet de mauvaises conditions travail et sont victimes d'abus. Les potentialités d'un développement économique durable seront affectées de façon négative si le gouvernement néglige de protéger juridiquement les droits des travailleurs informels.

En raison de la complexité et de la diversité des relations de travail informelles, tous les travailleurs informels ne sont pas pauvres et vulnérables. Il n'est donc pas aisé d'établir un constat unique. Les responsables politiques doivent développer des outils plus différenciés et plus sophistiqués pour cibler les différentes catégories de travailleurs qui composent l'économie informelle. La philosophie qui devrait animer les responsables politiques chinois à l'avenir concernant l'économie informelle mériterait d'être formulée en des termes « d'incorporation » et « de formalisation », c'est-à-dire d'élargissement des règlements au profit d'un cadre juridique plus équitable et humain.

49 Voir le texte intégral en anglais sur http://english.gov.cn/archive/publications/2016/09/29/ content_281475454482622.htm. 
Pour les raisons déjà citées, l'intervention règlementaire en Chine fait face à de nombreux défis. Les études empiriques sur les expériences chinoises montrent qu'une approche en douceur est peut-être plus appropriée à court terme qu'une approche très rigide ${ }^{50}$, dans une économie transitionnelle où l'efficacité et l'application de la loi doivent toutes deux être améliorées. Si les mesures règlementaires tendent à être plus contraignantes, les efforts législatifs doivent réduire au minimum les vides pour éviter de voir apparaître davantage de « conformité créative » et de contournements de la loi. En outre, afin d'assurer l'accès à la justice des travailleurs informels, il est nécessaire d'élargir les droits collectifs du travail, de renforcer le rôle des syndicats et des ONG, ainsi que d'intenter des actions collectives en cas de conflits du travail ${ }^{51}$.

50 Pour une description détaillée de l'approche douce et de l'approche dure, voir C. C. Williams and M. A. Lansky، "Informal Employment in Developed and Developing Economies: Perspectives and Policy Responses », International Labour Review, Vol. 152, 2013, n³-4, p. 368-371.

51 Ces dernières années, il y a eu davantage d'expériences d'expression collective des travailleurs informels en Chine. Par exemple, en 2007, le tout premier syndicat de travailleurs domestiques de Chine a été créé à Xi'an City. A Shanghai, les travailleurs migrants du secteur de la construction ont formé leurs syndicats sur les sites de construction. Dans les villes de Nanjing et Yantai, des travailleurs informels ont créé leurs syndicats communautaires. En même temps, au niveau micro, de plus en plus d'ONG ont été créés pour protéger les droits des travailleurs comme par exemple la Beijing's Female Peasants Family, les Hotline of Small Migrant Birds.

\section{WEI DAN}

Professeure titulaire, Vice-présidente de la Faculté de droit de l'Université de Macao.

Thèmes de recherche : droit du commerce international et des investissements, droit à la concurrence, arbitrage, droit de la consommation et droit du travail.

Parmi ses publications :

"Antitrust in China: An Overview of Recent Implementation of Anti-Monopoly Law", European Business Organization Law Review, Vol. 14, Issue 01, 2013, pp.119-139.

"Report of Macao Special Administrative Region", in M. Schlacher, The Prohibition of Age Discrimination in Labour Relations, Reports to the XVIII International Congress of Comparative Law Washington D.C., Nomos, Trier, Germany,2011, pp. 249-260. 